\title{
High degree of sex chromosome differentiation in stickleback fishes
}

\author{
Takahito Shikano*, Heini M Natri, Yukinori Shimada and Juha Merilä
}

\begin{abstract}
Background: Studies of closely related species with different sex chromosome systems can provide insights into the processes of sex chromosome differentiation and evolution. To investigate the potential utility of molecular markers in studying sex chromosome differentiation at early stages of their divergence, we examined the levels and patterns of genetic differentiation between sex chromosomes in nine-spined (Pungitius pungitius) and threespined sticklebacks (Gasterosteus aculeatus) using microsatellite markers.
\end{abstract}

Results: A set of novel microsatellite markers spanning the entire length of the sex chromosomes were developed for nine-spined sticklebacks using the sequenced genomes of other fish species. Sex-specific patterns of genetic variability and male-specific alleles were identified at most of these loci, indicating a high degree of differentiation between the $X$ and $Y$ chromosomes in nine-spined sticklebacks. In three-spined sticklebacks, male-specific alleles were detected at some loci confined to two chromosomal regions. In addition, male-specific null alleles were identified at several other loci, implying the absence of $Y$ chromosomal alleles at these loci. Overall, male-specific alleles and null alleles were found over a region spanning $81 \%$ of the sex chromosomes in three-spined sticklebacks.

Conclusions: High levels but distinct patterns of sex chromosome differentiation were uncovered in the stickleback species that diverged 13 million years ago. Our results suggest that the $\mathrm{Y}$ chromosome is highly degenerate in three-spined sticklebacks, but not in nine-spined sticklebacks. In general, the results demonstrate that microsatellites can be useful in identifying the degree and patterns of sex chromosome differentiation in species at initial stages of sex chromosome evolution.

\section{Background}

Recent advances in comparative genomic and molecular cytogenetic studies have greatly increased our understanding about the extent and pace of sex chromosome evolution [1,2]. For instance, therian and avian sex chromosomes are known to have originated independently from two different autosomal regions in a common ancestor $[1,3]$. In contrast, lower vertebrates exhibit a wide variety of sex chromosome and sex determination systems, implying multiple and independent origins of sex chromosomes [4-6]. Recent studies have shown that sex chromosomes of Oryzias fishes and sticklebacks (Gasterosteidae) have emerged 10 million years ago or even later [7-10]. Likewise, different heterogametic sex

\footnotetext{
* Correspondence: takahito.shikano@helsinki.fi

Ecological Genetics Research Unit, Department of Biosciences, University of
} Helsinki, P.O. Box 65, Fl-00014, Helsinki, Finland determination systems have been found in tilapia fishes and the frog Rana rugosa [11,12].

Despite the substantial progress in understanding the evolutionary history of sex chromosomes, there are still gaps in our knowledge about the process of sex chromosome differentiation. For instance, it has been hypothesized that sex chromosomes would typically evolve from a pair of autosomes that cease to recombine with each other after acquiring a sex determining role [13-15]. Suppression of recombination leads to further differentiation of the sex chromosomes and degeneration of the heterogametic chromosome (i.e. Y or W), resulting in morphologically differentiated sex chromosomes [14,16]. Accordingly, a heteromorphic sex chromosome pair is generally thought to have evolved through increasing stages of differentiation [14]. However, since chromosomal rearrangements can produce heteromorphism, even newly evolved sex chromosomes can be heteromorphic [2]. In addition, neither
C Biomed Central 
suppressed recombination nor heteromorphism always evolve in old-established sex chromosomes [2].

Studies of model organisms with young sex chromosomes have uncovered molecular characteristics at the initial stages of differentiation that align with theoretical expectations $[5,15,17]$. Yet, molecular differentiation of sex chromosomes has been assessed mainly from sequence analyses, which are not easily accomplished without access to sequenced genomes or equivalent genomic resources [17]. Thus, simple and accurate methods of evaluating sex chromosome differentiation in nonmodel organisms would be desirable. One potential approach is to use allelic variation in genetic markers. Since allelic differentiation at microsatellite loci is expected to be in a linear relationship with time since divergence $[18,19]$, microsatellite variation might be able to uncover the patterns and degree of sex chromosome differentiation. Indeed, although microsatellite markers have been rarely used for this purpose, they have been shown to be useful in detecting genetic differentiation between sex chromosomes in Drosophila [20]. Moreover, since a large proportion of microsatellite loci appear to be conserved in closely related species $[21,22]$, the use of microsatellite markers may also allow comparative genomic analyses in this context. Conversely, the sequenced genomes of closely related species can be used to predict microsatellite locations in the genome of a target species lacking the reference genome sequence [22]. An obvious limitation in the development of markers is that mutations in the regions flanking microsatellite loci make it difficult to develop primers that work in a target species. Yet, this problem can be circumvented by designing primers in conserved regions, which are identifiable using the genome sequences of multiple species in the same taxon. Accordingly, based on microsatellite locations in the genome of a related species, microsatellite markers can be developed for target chromosomes and genomic regions in other species lacking sequenced genomes.

Sticklebacks are widely used as model organisms in evolutionary biology [23,24]. The genome sequence of the three-spined stickleback (Gasterosteus aculeatus) provides a useful resource for studying the genetic basis of several phenotypic traits [25]. A sex determining region has been mapped to linkage group (LG) 19 in three-spined sticklebacks - a species possessing an XY sex chromosome system [8]. Their sex chromosomes are cytologically indistinguishable in the absence of molecular cytogenetic information [26]. In contrast, nine-spined sticklebacks (Pungitius pungitius) have a heteromorphic XY pair corresponding to three-spined stickleback LG 12 [10,27]. In particular, their sex chromosomes are characterized by a large $\mathrm{Y}$ chromosome which might be a result of a tandem duplication of the ancient Y chromosome or a duplication of an autosomal segment followed by insertion into the $\mathrm{Y}$ chromosome [28]. These different sex chromosome systems suggest their independent evolution in stickleback species [10]. Because of the rapid turnover of sex chromosome systems in closely related species, sticklebacks provide an ideal system to study the initial stages of sex chromosome differentiation $[8,10]$.

The aim of this study was to compare the level and patterns of genetic differentiation of sex chromosomes in nine-spined and three-spined sticklebacks using microsatellite markers. Since stickleback species exhibit a rapid turnover of sex chromosomes, their sex chromosomes are thought to be at the early stages of evolution. Therefore, we expected that the degree of genetic differentiation between sex chromosomes would be similar in nine-spined and three-spined sticklebacks. To facilitate the comparative genomic analyses, we devised a simple method for the development of microsatellite markers for target chromosomes and genomic regions in ninespined sticklebacks using the sequenced genomes of other fish species.

\section{Results}

\section{Nine-spined sticklebacks}

Twenty-three polymorphic markers, including 14 for LG 12 (Ppsm), were developed for nine-spined sticklebacks (Table 1). On average, 10.6 alleles per locus (range $=2-47$ ) were detected across the two populations (Table 1). The mean number of observed alleles and average expected heterozygosities were 10.3 and 0.66 in the Baltic Sea and 2.0 and 0.27 in Pyöreälampi, respectively (Additional file 1). Out of the 14 Ppsm loci, 10 were homozygous in all females and heterozygous in all males in the Pyöreälampi population (Figure 1, Additional file 1). Consequently, the number of observed alleles and expected and observed heterozygosities were significantly higher in males than females in this population (Wilcoxon signed rank test, $P<0.01$ for allele number, $P<0.001$ for heterozygosities). In the Baltic Sea population, observed heterozygosity was higher in males than females $(P<0.01$; Figure 1), but no significant differences were found in the number of alleles and expected heterozygosity $(P>0.05)$. While no loci showed significant $F_{\mathrm{IS}}$ in females, negative $F_{\text {IS }}$ was detected in males at 11 Ppsm loci in the Baltic Sea and at 13 Ppsm loci in Pyöreälampi (Figure 1, Additional file 1). Linkage disequilibrium was observed in all pairs of polymorphic Ppsm loci in Pyöreälampi, although 47 out of the 91 comparisons were not significant in the Baltic Sea (Additional file 2). In particular, linkage disequilibrium was not detected in several combinations including highly polymorphic loci (e.g. Ppsm3-5 and Ppsm14). The trend test identified significant associations between phenotypic sex and Ppsm loci in both populations (Additional file 3). All polymorphic Ppsm loci showed an association with sex in Pyöreälampi, whereas significant association was not 
Table 1 Location of 23 microsatellites in the three-spined stickleback genome and primer sequences for nine-spined sticklebacks

\begin{tabular}{|c|c|c|c|c|c|c|}
\hline \multirow[t]{2}{*}{ Locus } & \multicolumn{3}{|c|}{ Three-spined stickleback genome } & \multicolumn{3}{|c|}{ Microsatellite marker for nine-spined stickleback } \\
\hline & LG & Position (bp) & Repeat motif & Primer sequence $\left(5^{\prime}-3^{\prime}\right)$ & $A$ & Allele size (bp) \\
\hline \multirow[t]{2}{*}{ Ppsm1 } & $X \|$ & 188976 & $(\mathrm{CCT})_{6}$ & F: GGCTCTTCCGATGAGTTCTC & 6 & 298-308 \\
\hline & & & & R: GTCTGCGCGTCAGCATCC & & \\
\hline \multirow[t]{2}{*}{ Ppsm2 } & $X I I$ & 3625645 & $(\mathrm{AC})_{24}$ & F: GCCTCCCAGTCCTCTGT & 5 & $175-183$ \\
\hline & & & & R: GCCATGGAGTACGACATCATG & & \\
\hline \multirow[t]{2}{*}{ Ppsm3 } & $X I I$ & 4802750 & $(\mathrm{GT})_{16}$ & F: GAACGATGATTAATTTCACTC & 42 & $161-271$ \\
\hline & & & & R: CTGACCCTGACTGGAGG & & \\
\hline \multirow[t]{2}{*}{ Ppsm4 } & $X I I$ & 4843632 & $(C A)_{15}$ & F: CCAGCTGCTCTGTITTGTTAAC & 16 & $223-267$ \\
\hline & & & & R: CCTGGCCTCATTACAGTAAC & & \\
\hline \multirow[t]{2}{*}{ Ppsm5 } & $X I I$ & 5616109 & $(\mathrm{GAG})_{7}$ & F: ATCACGACTCTGAGGAGAG & 10 & $208-241$ \\
\hline & & & & R: TTCTTCAGCTCCACGGG & & \\
\hline \multirow[t]{2}{*}{ Ppsm6 } & XII & 7178391 & $(\mathrm{AC})_{12}$ & F: ATCGCCCTGCTGGTGGAG & 5 & $231-239$ \\
\hline & & & & R: GGAGCGCTGTTTCCGCC & & \\
\hline \multirow[t]{2}{*}{ Ppsm7 } & $X I I$ & 7695994 & $(\mathrm{GT})_{13}$ & F: GCAGCACTGTTGTCCAA & 9 & $73-143$ \\
\hline & & & & R: CTACTTGAACGATGCTC & & \\
\hline \multirow[t]{2}{*}{ Ppsm8 } & $X I I$ & 9015356 & $(\mathrm{GT})_{15}$ & F: CCAAAGGCAATTTCAAATCTC & 4 & 203-217 \\
\hline & & & & R: GAATGACAGGCTGTTTGTCTG & & \\
\hline \multirow[t]{2}{*}{ Ppsm9 } & $X \|$ & 10780938 & $(\mathrm{TG})_{16}$ & F: CAAGATGGACTACTCAAGG & 3 & $247-252$ \\
\hline & & & & R: CTATCAACCTCTCCAGCTTC & & \\
\hline \multirow[t]{2}{*}{ Ppsm10 } & XII & 12581231 & $(\mathrm{TG})_{7}$ & F: GCTTAGTGTTAATTGGTTCCTG & 7 & $218-232$ \\
\hline & & & & R: TAGACCCTGAGGGTGTG & & \\
\hline \multirow[t]{2}{*}{ Ppsm11 } & $X I I$ & 15639505 & $(\mathrm{AT})_{8}$ & F: AACAACGGTGCTATCTCCTCT & 7 & 186-198 \\
\hline & & & & R: TGGAATCCCATGCAGCGCAC & & \\
\hline \multirow[t]{2}{*}{ Ppsm12 } & $X \|$ & 16027500 & $(\mathrm{TG})_{8}$ & F: GCATGGTCATCATCTGGAG & 4 & $251-263$ \\
\hline & & & & R: ATGACACATGCATGGAGT & & \\
\hline \multirow[t]{2}{*}{ Ppsm13 } & $X I I$ & 16138995 & $(\mathrm{TG})_{13}$ & F: GCCTGCTACAAAGCTGA & 3 & $256-280$ \\
\hline & & & & R: CTTGAAGGACTCAAAGAAGCC & & \\
\hline \multirow[t]{2}{*}{ Ppsm14 } & $X I I$ & 18276705 & $(C A)_{11}$ & F: CCGATGGCCTGGTTCAC & 47 & $263-445$ \\
\hline & & & & R: GGCGTCATCATCACCGG & & \\
\hline \multirow[t]{2}{*}{ Pprm1 } & III & 4656850 & $(\mathrm{TG})_{15}$ & F: TGTGCTGCAGACCTCCAC & 5 & $202-214$ \\
\hline & & & & R: TGGCGTCACGGAGCTGAAG & & \\
\hline \multirow[t]{2}{*}{ Pprm2 } & IV & 62968 & $(C A)_{13}$ & F: GATACAGCTCCTGCTCCAG & 4 & 163-169 \\
\hline & & & & R: CCAGGATGAACCAGGTGAG & & \\
\hline \multirow[t]{2}{*}{ Pprm3 } & IV & 15800213 & $(\mathrm{GT})_{21}$ & F: GGCTTCTATTTCTGCCTCCC & 23 & 344-392 \\
\hline & & & & R: TACCTGAGCAGCTCGCAG & & \\
\hline \multirow[t]{2}{*}{ Pprm4 } & V & 4991466 & $(\mathrm{TGT})_{6}$ & F: GCTGGGCAGTATTCTGTGG & 7 & 288-306 \\
\hline & & & & R: AACATCCTCATCCACAGCAGC & & \\
\hline \multirow[t]{2}{*}{ Pprm5 } & $X V$ & 21776 & $(A C)_{16}$ & F: ATCCCAACGTCATCCAGCTC & 2 & 193-194 \\
\hline & & & & R: CAGCAGGAAGGTGTGCAG & & \\
\hline Pprm6 & $X V$ & 8323413 & $(C A)_{13}$ & F: CTGGAGCGTTTACAGGTGG & 8 & $364-382$ \\
\hline & & & & R: CTGCTGAGCTGAACAGGC & & \\
\hline Pprm7 & $X V I$ & 3587216 & $(A C)_{10}$ & F: CTGGAGACCAACAAGTTGAGG & 12 & $274-298$ \\
\hline & & & & R: CTTAACAAAGATCCTGCTGGACG & & \\
\hline Pprm8 & $X V I I I$ & 10203501 & $(\mathrm{GT})_{25}$ & F: CACCCATGTTCCTGTGCTTC & 11 & $306-380$ \\
\hline & & & & R: ACAAAGCCCTGCTCTCGAG & & \\
\hline Pprm9 & $X X$ & 5366535 & $(\mathrm{CA})_{14}$ & F: TCCTCATGATGTTGACCAGTGC & 3 & $166-172$ \\
\hline & & & & R: CTGGCCTATGGAAACCAGG & & \\
\hline
\end{tabular}

LG, linkage group. $\mathrm{F}$, forward; $\mathrm{R}$, reverse. $A$, number of observed alleles. 

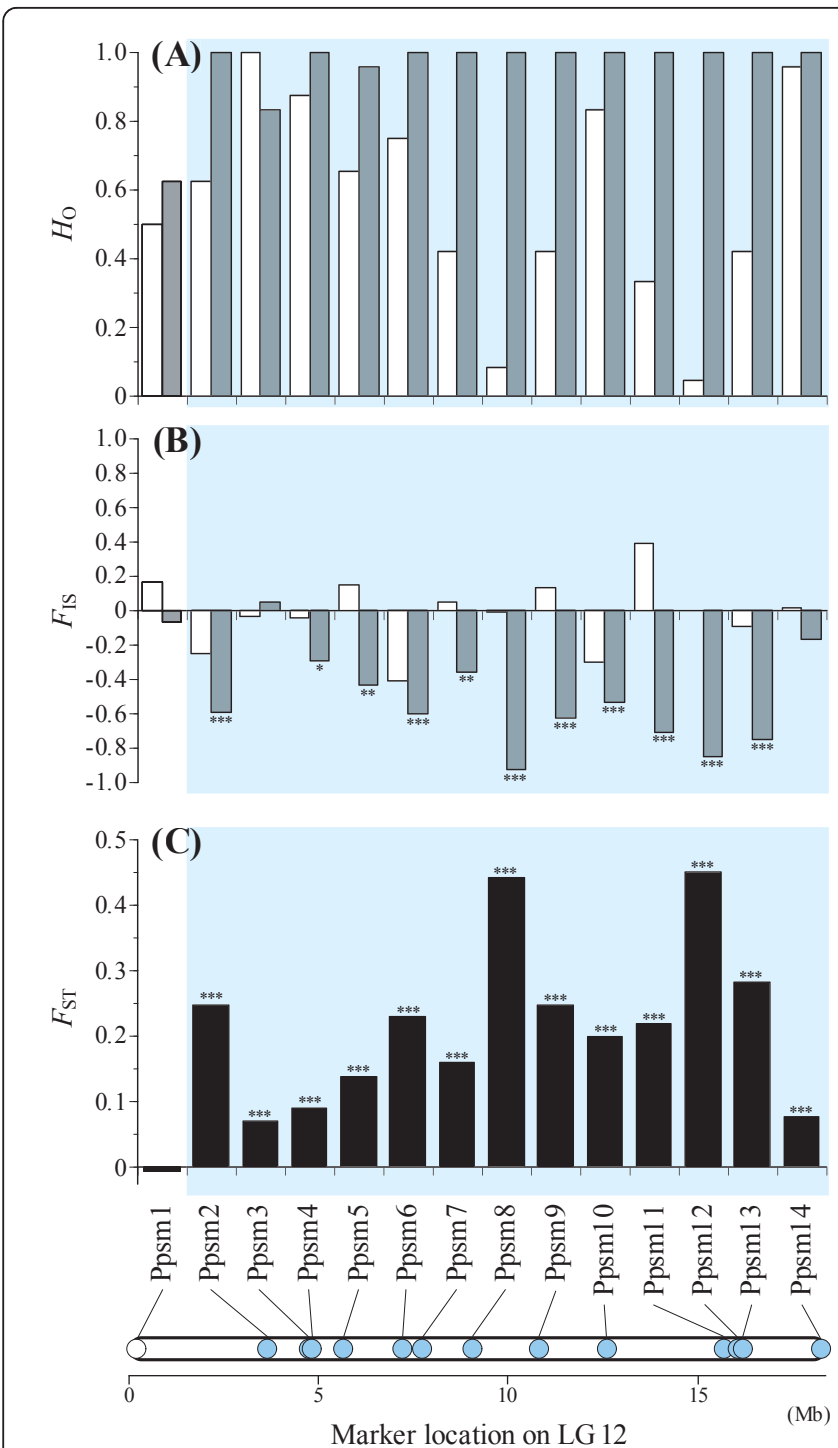
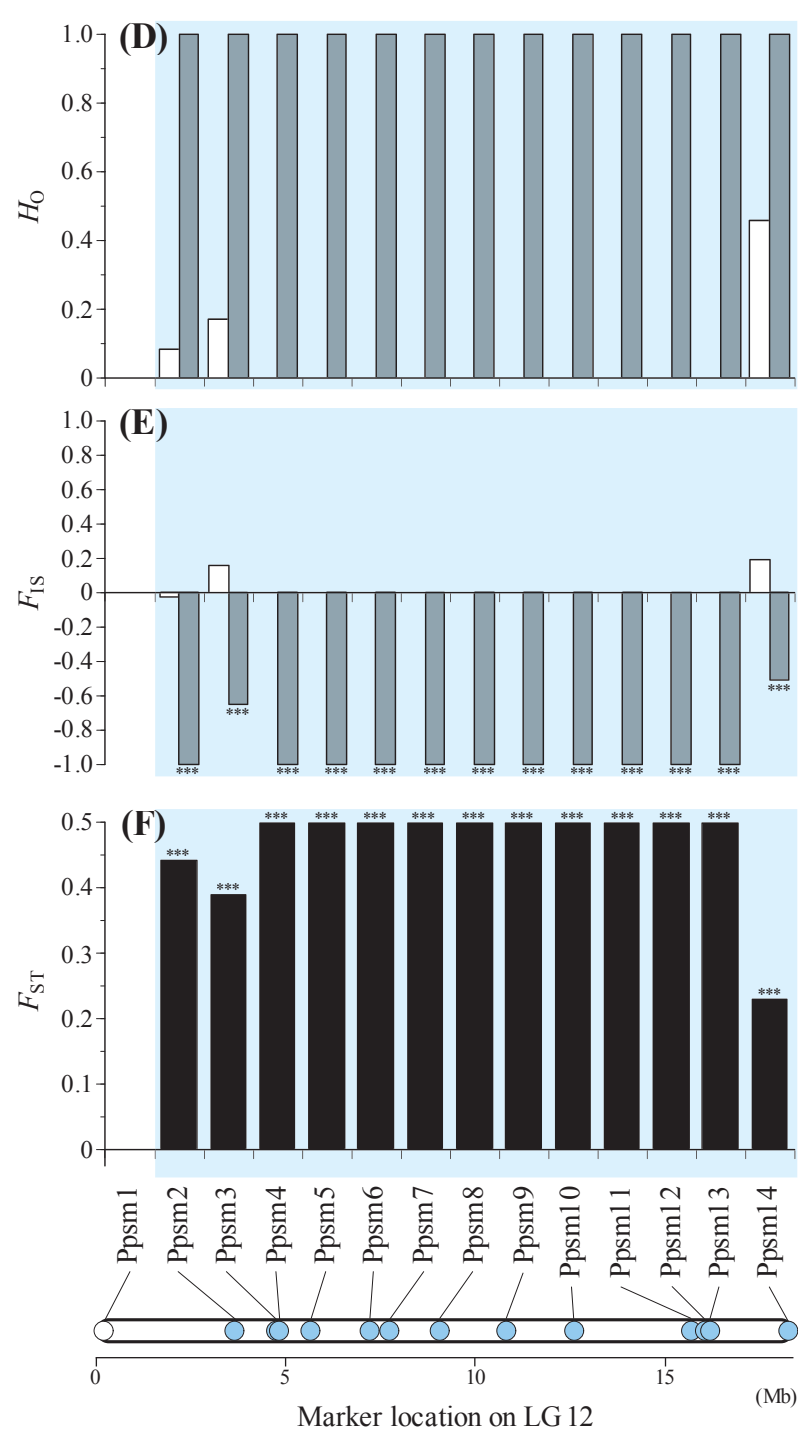

Figure 1 Observed heterozygosity $\left(H_{\mathrm{O}}\right), F_{\mathrm{IS}}$ and $F_{\mathrm{ST}}$ between females and males at 14 Ppsm loci in nine-spined sticklebacks. A and $\mathrm{D}$, $H_{\mathrm{O}}$ in females (white) and males (gray); $\mathbf{B}$ and $\mathbf{E}, F_{\text {IS }}$ in females (white) and males (gray); $\mathbf{C}$ and $\mathbf{F}$, $F_{\text {ST }}$ between females and males. A-C, Baltic Sea; D-F, Pyöreälampi. ${ }^{*} P<0.05$, ${ }^{* *} P<0.01$ and ${ }^{* *} P<0.001$. The blue colour indicates the loci where significant $F_{\text {ST }}$ was observed. Physical locations of loci were determined based on the three-spined stickleback genome.

observed for four Ppsm loci (Ppsm1, Ppsm3, Ppsm4 and Ppsm14) in the Baltic Sea (Additional file 3).

The analyses of genotypic frequencies at Ppsm loci identified several alleles that were observed only in males (Table 2). All males from Pyöreälampi possessed both male-specific and nonspecific alleles at each of the polymorphic loci (Table 2). No polymorphism was detected in the male-specific alleles in this population. Male-specific alleles were found at nine loci in all males of the Baltic Sea (Table 2). Although a male-specific allele was not detected at Ppsm5, the same allele (229) was observed in most (23 out of 24) males and a few (2 out of 24) females. $F_{\mathrm{ST}}$ values between females and males were significant for 13 Ppsm loci in both populations (Figure 1). For these loci, $F_{\mathrm{ST}}$ values were larger in the Pyöreälampi than in the Baltic Sea.

\section{Three-spined sticklebacks}

A total of 161 alleles were observed at 14 loci, with an average of 11.5 alleles per locus (range $=2-31$ ) in threespined sticklebacks (Additional files 4 and 5). Linkage disequilibrium was observed in only eight out of the 91 comparisons (Additional file 6). Expected heterozygosity ranged from 0.39 to 0.93 among the loci with a mean of 0.70 (Additional file 5). Several loci showed different levels of observed heterozygosity between females and 
Table 2 Male-specific alleles at 14 Ppsm loci in two populations of nine-spined sticklebacks

\begin{tabular}{lllll}
\hline Locus & \multicolumn{3}{l}{ Baltic Sea } & Pyöreälampi \\
\cline { 2 - 5 } & Male-specific allele* & Other allele & Male-specific allele* & Other allele \\
\hline Ppsm1 & - & $298-308$ & - & - \\
Ppsm2 & $179(100.0)$ & $175-181$ & $179(100.0)$ & $181-183$ \\
Ppsm3 & $171(54.2)$ & $161-271$ & $191(100.0)$ & $209-215$ \\
Ppsm4 & $247(70.8)$ & $223-267$ & $247(100.0)$ & 227 \\
Ppsm5 & - & $208-241$ & $229(100.0)$ & 235 \\
Ppsm6 & $232(100.0)$ & $231-239$ & $232(100.0)$ & 75 \\
Ppsm7 & $125-143(100.0)$ & $73-77$ & $141(100.0)$ & 215 \\
Ppsm8 & $203(100.0)$ & $211-217$ & $203(100.0)$ & 252 \\
Ppsm9 & $247(100.0)$ & $251-252$ & $247(100.0)$ & 220 \\
Ppsm10 & $222(100.0)$ & $218-232$ & $222(100.0)$ & 188 \\
Ppsm11 & $197(100.0)$ & $186-198$ & $197(100.0)$ & 257 \\
Ppsm12 & $251-253(100.0)$ & $257-263$ & $253(100.0)$ & 256 \\
Ppsm13 & $280(100.0)$ & $256-258$ & $280(100.0)$ & $281-287$
\end{tabular}

*The percentage of males having the allele(s) is indicated in parentheses. Minor alleles (< 15\%) are not shown except for Ppsm7 and Ppsm12.

males (Figure 2, Additional file 5). Although no loci showed significant $F_{\mathrm{IS}}$ in females, 12 loci exhibited significant $F_{\text {IS }}$ in males (Figure 2, Additional file 5). Out of these 12 loci, five were heterozygous in all males, showing negative $F_{\mathrm{IS}}$. At these loci, which were located in two chromosomal regions (i.e. 3.2-4.0 $\mathrm{Mb}$ and 9.4-11.8 Mb), male-specific alleles were detected in all males in heterozygous states (Table 3 ). In contrast, although two to 20 alleles were observed for each locus, no heterozygous males were found at seven loci that showed positive $F_{\mathrm{IS}}$ (Figure 2, Additional file 5). No male-specific alleles were identified at these loci (Table 3). MICRO-CHECKER analyses indicated the presence of null alleles at the seven loci in males, but not in females. These loci were located sequentially in two separate chromosomal regions (i.e. 5.1-7.4 Mb and 14.7-19.6 Mb) of LG 19 (Figure 2, Additional file 4).

Significant associations between phenotypic sex and loci were detected for the five loci that possessed male-specific alleles (Additional file 7). $F_{\mathrm{ST}}$ between females and males was significant for these, but not for the other loci (Figure 2).

\section{Discussion}

Our study uncovered sex-specific patterns of genetic variability and male-specific alleles at several loci in both stickleback species. In addition, male-specific null alleles were identified at several loci in three-spined sticklebacks, suggesting the absence of $Y$ chromosomal alleles at these loci. The high allelic heterogeneity between sexes indicates high levels of sex chromosome differentiation, which likely reflects recombination suppression between these chromosomes. In the following, we discuss these issues, as well as our approach to develop microsatellite markers in a non-model organism.

\section{Differentiation of stickleback sex chromosomes}

Our results indicate that 13 markers (Ppsm2-14) are linked to each other, and are associated with phenotypic sex in nine-spined sticklebacks. These indications were more robust in a population with low genetic diversity (Pyöreälampi) than in one with high genetic diversity (Baltic Sea). Indeed, all males of Pyöreälampi possessed one particular male-specific allele for each of the 13 loci. Given the male heterogametic inheritance of this species [28], these alleles should be located on the Y chromosome. Accordingly, only one Y chromosome haplotype was identified in this population. In contrast, three out of the 13 loci did not exhibit an association with phenotypic sex in the Baltic Sea population. Since these three loci were highly polymorphic, this could be due to their high mutation rates. Even in this highly variable population, the male-specific allele was monomorphic at seven loci. However, polymorphisms were detected in the remaining alleles, which are putatively located on the $\mathrm{X}$ chromosome. The different level of polymorphism between the sex chromosomes is explainable by the fact that the effective population size of the $\mathrm{Y}$ chromosome is one-third of that of the $\mathrm{X}$ chromosome [29]. In general, our results indicate that sex chromosomes are highly differentiated throughout most of their length in nine-spined sticklebacks.

Based on karyotype analyses, the Y chromosome of nine-spined sticklebacks is characterized by a much larger size than the other chromosomes [10,28]. Because none of the autosomes or autosomal arms are missing, the additional segment observed in the $\mathrm{Y}$ chromosome is 

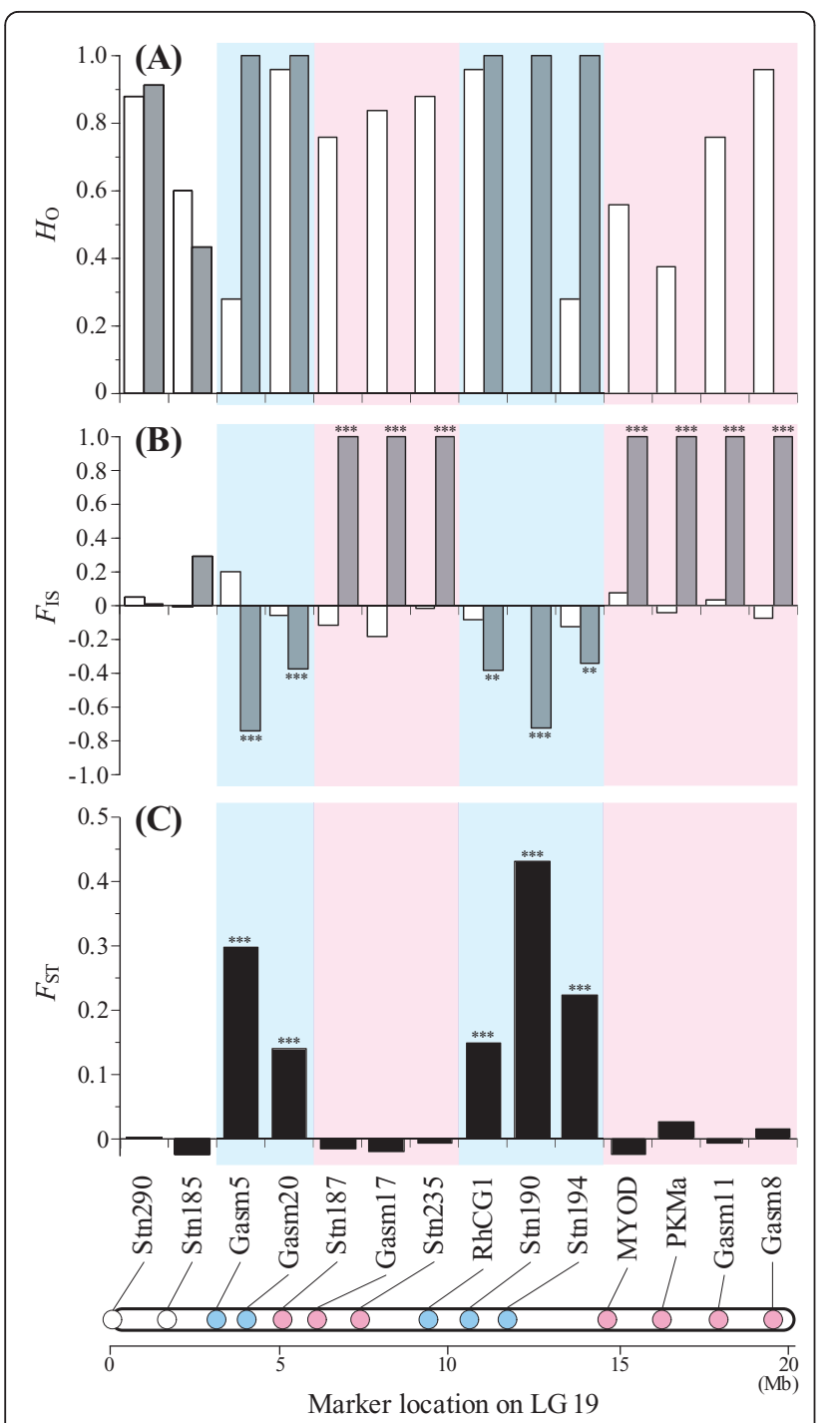

Figure 2 Observed heterozygosity $\left(H_{\mathrm{O}}\right), F_{\mathrm{IS}}$ and $F_{\mathrm{ST}}$ between females and males at 14 loci in three-spined sticklebacks. $\mathbf{A}, H_{O}$ in females (white) and males (gray); $\mathbf{B}, F_{15}$ in females (white) and males (gray); $\mathbf{C}, F_{\text {ST }}$ between females and males. ${ }^{*} P<0.01$ and ${ }^{* * *} P$ $<0.001$. The blue colour indicates the loci where significant $F_{\text {ST }}$ was observed, and the red colour represents the loci where no heterozygous males were found. Physical locations of loci were determined based on the three-spined stickleback genome but modified according to Ross and Peichel [26].

assumed to have originated from a tandem duplication of the ancient $\mathrm{Y}$ chromosome or a duplication of an autosomal segment followed by insertion into the $Y$ chromosome [28]. Although we examined allelic variation along the sex chromosomes with several markers - for which primers were developed in conserved sequences of divergent species - no loci showed the patterns of multilocus amplification which can be an indication of the Y chromosome duplication. Thus, an extra segment of the $Y$ chromosome is more likely to be derived from a duplication of an autosome than that of the $\mathrm{Y}$ chromosome.

In three-spined sticklebacks, an allozyme of isocitrate dehydrogenase $(I D H)$ has been found to be associated with phenotypic sex $[30,31]$. In accordance with this observation, a linkage mapping analysis showed that the sex determining region is located near the $I D H$ gene of LG 19 [8]. In our study, male-specific alleles, which should be located on the $\mathrm{Y}$ chromosome, were identified at five loci. Indeed, three of these loci were closely linked to the $I D H$ gene $(11.3 \mathrm{Mb})$. However, the remaining two loci were found in a chromosomal region (3.2-4.0 Mb) far from the $I D H$ gene, implying that there is extensive differentiation. In addition, male-specific null alleles were identified at several loci located in two different chromosomal regions. This lack of heterozygous males suggests that amplification of Y chromosomal alleles is absent at these loci. This could be due to complete deletion of the microsatellite loci or polymorphisms in the primer binding sites of these loci in the Y chromosome. Overall, $\mathrm{Y}$ specific alleles and null alleles were identified over 16.4 $\mathrm{Mb}$ of a chromosomal region (3.2-19.6 Mb), which corresponds to $81 \%$ of the $\mathrm{X}$ chromosome. A large deletion of the $Y$ chromosome was also suggested by a previous study using fluorescence in situ hybridization [26]. Indeed, the deletion was assumed to be present in one of the regions where $\mathrm{Y}$ chromosomal null alleles were identified (i.e. 14.7-19.6 Mb). Other Y chromosomal null alleles were found in the region where multiple inversions appear to have occurred [26]. It has also been demonstrated by sequence analysis that the Y chromosome exhibits substantial nucleotide divergence from the homologous region on the $\mathrm{X}$ chromosome mainly due to multiple insertions and deletions [8]. Together with these studies, our results suggest that the Y chromosome is highly degenerate in three-spined sticklebacks. Since closely related stickleback species exhibit the rapid turnover of sex chromosomes and sex determination systems, their sex chromosomes are thought to be at the early stages of evolution, as is generally observed in lower vertebrates $[8,10]$. Our study uncovered high levels but distinct patterns of sex chromosome differentiation in closely related stickleback species, which diverged about 13 million years ago [32]. In contrast to three-spined sticklebacks, we did not detect signatures of Y chromosome degeneration in the nine-spined stickleback despite the apparent sex chromosome heteromorphy in this species [28]. Therefore, it is unlikely that the Y chromosome of the nine-spined stickleback has degenerated as much as that of the three-spined stickleback.

Sexually antagonistic selection is expected to facilitate the suppression of recombination between sex chromosomes, and thus assumed to be the primary driving force 
Table 3 Male-specific alleles at $\mathbf{1 4}$ loci in three-spined sticklebacks

\begin{tabular}{lll}
\hline Locus & \multicolumn{2}{l}{ Baltic Sea } \\
\cline { 2 - 3 } & Male-specific allele* & Other allele \\
\hline Stn290 & - & $100-178$ \\
Stn185 & - & $152-160$ \\
Gasm5 & $82(100.0)$ & $85-89$ \\
Gasm20 & $191(100.0)$ & $313-371$ \\
Stn187 & - & $156-184$ \\
Gasm17 & - & $193-213$ \\
Stn235 & - & $127-155$ \\
RhCG1 & $254(100.0)$ & $241-275$ \\
Stn190 & $231-279(100.0)$ & 183 \\
Stn194 & $123-129(100.0)$ & $85-91$ \\
MYOD & - & $150-162$ \\
PKMa & - & $269-271$ \\
Gasm11 & - & $424-456$ \\
Gasm8 & - & $376-406$ \\
\hline
\end{tabular}

*The percentage of males having the allele(s) is indicated in parentheses. Minor alleles $(<15 \%)$ are not shown except for Stn194 and Stn190.

behind sex chromosome differentiation [14]. Additionally, chromosome rearrangements, such as inversions, translocations and centric fusions involving an autosome and a sex chromosome, are known to reduce or suppress crossing over in the regions around the breakpoints in heterozygotes with the standard arrangement [14]. Such rearrangements can create linkage between sexually antagonistic genes and sex chromosomes, and thereby can be favored by natural selection $[14,33]$. As such, the high genetic divergence between the sex chromosomes of nine-spined and three-spined sticklebacks could be a result of both sexually antagonistic selection and chromosome rearrangements. Further research needs to be focused on the role of sexually antagonistic selection in sex chromosome differentiation in these species. In addition, it should be interesting to investigate intraspecific variation of sex chromosome differentiation and rearrangements among genetically divergent populations.

\section{Marker development for non-model organisms}

To facilitate comparative genomic analyses, we devised a simple method for the development of microsatellite markers at target chromosomes and genomic regions using the sequenced genomes of other species. Our approach is similar to cross-species transfer of microsatellite markers, which can be performed without sequence information from a target species, but is different in the sense that conserved microsatellite flanking regions in divergent species are used as primer binding sites. Thus, it is possible to minimize amplification failures, which are a general concern in the cross-species utility of microsatellite markers [34]. In fact, when primers are designed using three-spined stickleback sequences alone, amplification success in ninespined sticklebacks is low (28.6\%) [22]. Moreover, our approach has an advantage over traditional cross-species amplification approaches because microsatellite markers can be developed for specific chromosomes and genomic regions based on the sequenced genome of other species. Furthermore, since primers are developed for conserved sequences in divergent species, these markers should be useful also in other stickleback species. Therefore, our approach for the development of microsatellite markers can facilitate comparative genomic analyses of stickleback species. In light of the rapidly increasing numbers of sequenced genomes and genomic resources, molecular tools for species lacking sequenced genomes can be developed using available genomic information of other species, as demonstrated in this study.

\section{Conclusions}

To summarize, we uncovered high levels of sex chromosome differentiation in two stickleback species, suggesting that sex chromosomes have rapidly differentiated from each other. Our results further imply that the Y chromosome is highly degenerate in three-spined sticklebacks, but not in nine-spined sticklebacks. In general, our study demonstrates that microsatellites can be useful in identifying the degree and patterns of sex chromosome differentiation. Further comparative genomic analyses within and between stickleback species - possibly with the aid of the approach for the development of molecular markers described in this study - should facilitate our understanding of the evolutionary mechanisms underlying sex chromosome differentiation.

\section{Methods}

\section{Fish samples}

Mature nine-spined sticklebacks were collected from the Baltic Sea $\left(60^{\circ} 12^{\prime} \mathrm{N}, 25^{\circ} 11^{\prime}\right.$ E) and from Pyöreälampi pond $\left(66^{\circ} 16^{\prime} \mathrm{N}, 29^{\circ} 26^{\prime} \mathrm{E}\right)$ in 2008 . These populations were selected to cover different levels of genetic diversity (average heterozygosity at 12 microsatellite loci, $H_{\mathrm{E}}=$ 0.590 in Baltic Sea and $H_{\mathrm{E}}=0.004$ in Pyöreälampi) [35], which can influence the identification of sex chromosome differentiation using microsatellite markers. Threespined sticklebacks were sampled from the Baltic Sea $\left(60^{\circ} 12^{\prime} \mathrm{N}, 2^{\circ} 11^{\prime} \mathrm{E}\right)$ in 2008 . Phenotypic sex was determined by examining gonads after the fish had been anesthetised with an overdose of MS-222 (tricane methanesulphonate). All procedures were performed under license from the Animal Experiment Board in Finland (ELLA; STH379A).

\section{Microsatellite primer development}

Using the three-spined stickleback genome [36], microsatellites were surveyed in regions randomly chosen 
over a wide range of LG 12 (Table 1), which corresponds to the sex chromosomes in nine-spined sticklebacks [10,27]. To design primers for nine-spined sticklebacks, conserved regions were searched in microsatellite flanking regions by aligning sequences of the three-spined stickleback and medaka [37]. Based on the location of microsatellites and conserved regions, primer sequences were designed manually, targeting appropriate melting temperature and GC content (Table 1). In addition to LG 12, some primers were developed for other linkage groups to verify variability of marker loci on sex chromosomes and autosomes (Table 1). For three-spined sticklebacks, 14 microsatellites covering a wide range of the sex chromosomes of this species (LG 19) were selected based on the sequenced genome [36] (Additional file 4). Of these, nine markers were previously reported $[38,39]$, and new primers were developed for the remaining five loci with the genome sequences using WebSat [40]. Since the genome sequences were obtained from a female specimen [25], the novel primers were assumed to be designed based on the $\mathrm{X}$ chromosome sequences. All novel microsatellite primers were deposited in the National Center for Biotechnology Information (NCBI) Probe Database [PUIDs: 10552794-10552816, 1070123210701236].

\section{Microsatellite genotyping}

Total DNA was extracted from fin clips using a silicafine based purification [41] following proteinase K digestion. Each forward primer was labelled with a fluorescent dye (FAM, HEX or TET), and the 5'-end of each reverse primer was modified with a GTTT-tail [42]. For efficient screening, PCRs were conducted using the Qiagen Multiplex PCR Kit (Qiagen) in $10 \mu$ l reaction volumes containing $1 \times$ Multiplex PCR Master Mix, 0.5× Q-Solution, 2 pmol of each primer and 10-20 ng of template DNA. The reactions were performed by the following cycle: an initial activation step at $95^{\circ} \mathrm{C}$ for $15 \mathrm{~min}$, followed by $30 \mathrm{~s}$ at $94^{\circ} \mathrm{C}, 90 \mathrm{~s}$ at $53^{\circ} \mathrm{C}$ and $60 \mathrm{~s}$ at $72^{\circ} \mathrm{C}$ for 30 cycles with a final extension at $60^{\circ} \mathrm{C}$ for $5 \mathrm{~min}$. PCR products were visualized with a MegaBACE 1000 automated sequencer (Amersham Biosciences) and their sizes were determined with ET-ROX 550 size standard (Amersham Biosciences). Polymorphism was initially screened using 24 individuals (12 females and 12 males) of nine-spined or three-spined sticklebacks from the Baltic Sea. For the identified polymorphic loci, a total of 48 individuals (24 females and 24 males for nine-spined sticklebacks and 25 females and 23 males for three-spined sticklebacks) were genotyped in each population. Alleles were scored using Fragment Profiler 1.2 (Amersham Biosciences) with visual inspection and manual corrections of alleles.

\section{Data analyses}

Locus specific heterozygosity and $F_{\text {IS }}$ were calculated using FSTAT 2.9.3 [43]. The significance of $F_{\text {IS }}$ was assessed by 10000 permutations. For marker loci located on sex chromosomes, $F_{\mathrm{IS}}$ is expected to be lower in heterogametic males than in homogametic females if alleles are not shared between the sex chromosomes. Linkage disequilibrium was tested between pairs of loci in each population. An association between phenotypic sex and alleles at marker loci was investigated using the trend test $[44,45]$ as implemented in PowerMarker 3.25 [46]. To identify Y chromosome specific alleles, genotypic frequencies and allele distributions were compared between males and females. In addition, to evaluate genetic differentiation between sex chromosomes, $F_{\mathrm{ST}}$ was estimated between females and males using the method of Weir and Cockerham [47] as implemented in GENEPOP 4.0 [48]. Statistical significance of $F_{\mathrm{ST}}$ values was determined using 10000 permutations. In three-spined sticklebacks, some polymorphic loci appeared to be homozygous in all males (see Results). For these loci, the presence of sexspecific null alleles was tested using MICRO-CHECKER [49]. Since a large proportion of microsatellites are conserved between three-spined and nine-spined sticklebacks [22,27], microsatellite locations on LG 12 in ninespined sticklebacks were estimated based on the threespined stickleback genome. Based on the fact that the orientation of supercontig 3 of LG 19 in Ensembl is inverted as compared to the genetic map [26], marker locations on LG 19 were determined by reversing the sequence of this supercontig according to Ross and Peichel [26] (Additional file 4). Sequential Bonferroni corrections [50] were applied for all multiple comparisons to minimize type I errors.

\section{Additional material}

Additional file 1: Number of observed alleles $(A)$, observed and expected heterozygosities $\left(H_{\mathrm{O}}\right.$ and $\left.H_{\mathrm{E}}\right)$ and $F_{\mathrm{IS}}$ at 23 loci in two populations of nine-spined sticklebacks.

Additional file 2: Significance of linkage disequilibrium among 23 loci in the Baltic Sea (below the diagonal) and Pyöreälampi (above the diagonal) of nine-spined sticklebacks.

Additional file 3: Association between phenotypic sex and loci in two populations of nine-spined sticklebacks.

Additional file 4: Location of 14 microsatellites and primer sequences for three-spined sticklebacks

Additional file 5: Number of observed alleles $(A)$, observed and expected heterozygosities $\left(H_{\mathrm{O}}\right.$ and $\left.H_{\mathrm{E}}\right)$ and $F_{\mathrm{IS}}$ at 14 loci in threespined sticklebacks.

Additional file 6: Significance of linkage disequilibrium among 14 loci in three-spined sticklebacks

Additional file 7: Association between phenotypic sex and loci in three-spined sticklebacks. 


\section{Acknowledgements}

We thank Abigel Gonda, John Loehr and people at the Oulanka Research Station, for help in obtaining samples. Thanks are also due to Chikako Matsuba for comments on an earlier version of this manuscript and Jacquelin DeFaveri for proofreading. Our study was supported by the Academy of Finland (JM, TS) and the Japan Society for the Promotion of Science (TS, YS).

\section{Authors' contributions}

TS conceived of the study, performed the molecular work, conducted the data analyses and wrote the manuscript. HMN participated in the molecular work. YS collected the fish samples and assisted with the laboratory work. JM contributed to writing the manuscript. All authors read and approved the final manuscript.

Received: 7 March 2011 Accepted: 29 September 2011 Published: 29 September 2011

\section{References}

1. Ezaz T, Stiglec R, Veyrunes F, Graves JAM: Relationships between vertebrate ZW and XY sex chromosome systems. Curr Biol 2006, 16 R736-R743.

2. Charlesworth D, Mank JE: The birds and the bees and the flowers and the trees: Lessons from genetic mapping of sex determination in plants and animals. Genetics 2010, 186:9-31.

3. Veyrunes F, Waters PD, Miethke P, Rens W, McMillan D, Alsop AE, Grützner F, Deakin JE, Whittington CM, Schatzkamer K, Kremitzki CL, Graves T, Ferguson-Smith MA, Warren W, Marshall Graves JA: Bird-like sex chromosomes of platypus imply recent origin of mammal sex chromosomes. Genome Res 2008, 18:965-973.

4. Mank JE, Promislow DEL, Avise JC: Evolution of alternative sexdetermining mechanisms in teleost fishes. Biol J Linn Soc 2006, 87:83-93.

5. Volff JN, Nanda I, Schmid M, Shartl M: Governing sex determination in fish: regulatory putsches and ephemeral dictators. Sex Dev 2007, 2007:85-99.

6. Wilson MA, Makova KD: Genomic analyses of sex chromosome evolution. Annu Rev Genomics Hum Genet 2009, 10:333-354.

7. Kondo M, Nanda I, Hornung U, Schmid M, Schartl M: Evolutionary origin of the medaka Y chromosome. Curr Biol 2004, 14:1664-1669.

8. Peichel CL, Ross JA, Matson CK, Dickson M, Grimwood J, Schmutz J, Myers RM, Mori S, Schluter D, Kingsley DM: The master sex-determination locus in threespine sticklebacks is on a nascent y chromosome. Curr Biol 2004, 14:1416-1424.

9. Tanaka K, Takehana Y, Naruse K, Hamaguchi S, Sakaizumi M: Evidence for different origins of sex chromosomes in closely related Oryzias fishes: substitution of the master sex-determining gene. Genetics 2007, 177:2075-2081

10. Ross JA, Urton JR, Boland J, Shapiro MD, Peichel CL: Turnover of sex chromosomes in the stickleback fishes (Gasterosteidae). PLoS Genet 2009, 5:e1000391.

11. Cnaani A, Lee B-Y, Zilberman N, Ozouf-Costaz C, Hulata G, Ron M, D'Hont A, Baroiller J-F, D'Cotta H, Penman DJ, Tomasino E, Coutanceau J-P, Pepey E, Shirak A, Kocher TD: Genetics of sex determination in tilapiine species. Sex Dev 2008, 2:43-54.

12. Miura I: An evolutionary witness: the frog Rana rugosa underwent change of heterogametic sex from XY male to ZW female. Sex Dev 2007, 1:323-331.

13. Schartl M: Sex chromosome evolution in non-mammalian vertebrates. Curr Opin Genet Dev 2004, 14:634-641.

14. Charlesworth D, Charlesworth B, Marais G: Steps in the evolution of heteromorphic sex chromosomes. Heredity 2005, 95:118-128.

15. Bachtrog A: A dynamic view of sex chromosome evolution. Curr Opin Genet Dev 2006, 16:578-585.

16. Charlesworth $B$, Charlesworth D: The degeneration of $Y$ chromosomes. Phil Trans R Soc Lond B 2000, 355:1563-1572.

17. Ming R, Moore PH: Genomics of sex chromosomes. Curr Opin Plant Biol 2007, 10:123-130.

18. Goldstein DB, Ruíz-Linares A, Feldman M, Cavalli-Sforza LL: Genetic absolute dating based on microsatellites and the origin of modern humans. Proc Natl Acad Sci USA 1995, 92:6720-6727.
19. Sun JX, Mullikin JC, Patterson N, Reich DE: Microsatellites are molecular clocks that support accurate inferences about history. Mol Biol Evol 2009, 26:1017-1027.

20. Schlötterer C: Microsatellite analysis indicates genetic differentiation of the neo-sex chromosomes in Drosophila americana americana. Heredity 2000, 85:610-616.

21. Vowles EJ, Amos W: Quantifying ascertainment bias and species-specific length differences in human and chimpanzee microsatellites using genome sequences. Mol Biol Evol 2006, 23:598-607.

22. Shikano T, Ramadevi J, Shimada Y, Merilä J: Utility of sequenced genomes for microsatellite marker development in non-model organisms: a case study of functionally important genes in nine-spined sticklebacks (Pungitius pungitius). BMC Genomics 2010, 11:334.

23. Bell MA, Foster SA: Introduction to the evolutionary biology of the threespine stickleback. In The Evolutionary Biology of the Threespine Stickleback. Edited by: Bell MA, Foster SA. Oxford: Oxford University Press; 1994:1-27.

24. Östlund-Nilsson S, Mayer I: The biology of other sticklebacks. In Biology of the Three-Spined Stickleback. Edited by: Östlund-Nilsson S, Mayer I, Huntingford FA. Boca Raton: CRC Press; 2007:353-372.

25. Kingsley $\mathrm{DM}$, Peichel $\mathrm{CL}$ : The molecular genetics of evolutionary change in sticklebacks. In Biology of the Three-Spine Stickleback. Edited by: ÖstlundNilsson S, Mayer I, Huntingford FA. Boca Raton: CRC Press; 2007:41-81.

26. Ross JA, Peichel CL: Molecular cytogenetic evidence of rearrangements on the $Y$ chromosome of threespine stickleback fish. Genetics 2008, 179:2173-2182.

27. Shapiro MD, Summers BR, Balabhadra S, Aldenhoven JT, Miller AL, Cunningham C, Bell MA, Kingsley DM: The genetic architecture of skeletal convergence and sex determination in ninespine sticklebacks. Curr Biol 2009, 19:1140-1145.

28. Ocalewicz K, Fopp-Bayat D, Woznicki P, Jankun M: Heteromorphic sex chromosomes in the ninespine stickleback Pungitius pungitius. J Fish Biol 2008, 73:456-462.

29. Jobling MA, Tyler-Smith C: The human Y chromosome: an evolutionary marker comes of age. Nat Rev Genet 2003, 4:598-612.

30. Avise JC: Genetics of plate morphology in an unusual of threespine sticklebacks (Gasterosteus aculeatus). Genet Res 1976, 27:33-46.

31. Withler RE, MCPhail JD: Genetic variability in freshwater and anadromous sticklebacks (Gasterosteus aculeatus) of Southern British Columbia. Can J Zool 1985, 63:528-533.

32. Bell MA, Stewart JD, Park PJ: The world's oldest fossil threespine stickleback fish. Copeia 2009, 2009:256-265.

33. Charlesworth $D$, Charlesworth B: Sex differences in fitness and selection for centric fusions between sex-chromosomes and autosomes. Genet Res 1980, 35:205-214.

34. Barbará T, Palma-Silva C, Paggi GM, Bered F, Fay MF, Lexer C: Cross-species transfer of nuclear microsatellite markers: potential and limitations. Mol Ecol 2007, 16:3759-3767.

35. Shikano T, Shimada Y, Herczeg G, Merilä J: History vs. habitat type: explaining the genetic structure of European nine-spined stickleback (Pungitius pungitius) populations. Mol Ecol 2010, 19:1147-1161.

36. Ensembl Stickleback. [http://www.ensembl.org/Gasterosteus_aculeatus/ Info/Index].

37. Ensembl Medaka. [http://www.ensembl.org/Oryzias_latipes/Info/Index].

38. Peichel CL, Nereng K, Ohgi KA, Cole BLE, Colosimo PF, Buerkle CA, Schluter D, Kingsley DM: The genetic architecture of divergence between threespine stickleback species. Nature 2001, 414:901-905.

39. Shimada Y, Shikano T, Merilä J: A high incidence of selection on physiologically important genes in the three-spined stickleback, Gasterosteus aculeatus. Mol Biol Evol 2011, 28:181-193.

40. Martins WS, Lucas DCS, Neves KFS, Bertioli DJ: WebSat - A web software for microsatellite marker development. Bioinformation 2009, 3:282-283.

41. Elphinstone MS, Hinten GN, Anderson MJ, Nock CJ: An inexpensive and high-throughput procedure to extract and purify total genomic DNA for population studies. Mol Ecol Notes 2003, 3:317-320.

42. Brownstein MJ, Carpten JD, Smith JR: Modulation of non-templated nucleotide addition by Taq DNA polymerase: primer modifications that facilitate genotyping. BioTechniques 1996, 20:1004-1010.

43. Goudet J: FSTAT (Version 1.2): a computer program to calculate Fstatistics. J Hered 1995, 86:485-486. 
44. Armitage P: Tests for linear trends in proportions and frequencies. Biometrics 1955, 11:375-386.

45. Slager SL, Schaid DJ: Evaluation of candidate genes in case-control studies: a statistical method to account for related subjects. Am J Hum Genet 2001, 68:1457-1462.

46. Liu K, Muse SV: PowerMarker: an integrated analysis environment for genetic marker analysis. Bioinformatics 2005, 21:2128-2129.

47. Weir BS, Cockerham CC: Estimating F-statistics for the analysis of population structure. Evolution 1984, 38:1358-1370.

48. Rousset F: Genepop'007: a complete reimplementation of the Genepop software for Windows and Linux. Mol Ecol Resour 2008, 8:103-106.

49. Van Oosterhout C, Hutchinson WF, Wills DP, Shipley P: MICRO-CHECKER: software for identifying and correcting genotyping errors in microsatellite data. Mol Ecol Notes 2004, 4:535-538.

50. Rice WR: Analyzing tables of statistical tests. Evolution 1989, 43:223-225.

doi:10.1186/1471-2164-12-474

Cite this article as: Shikano et al: High degree of sex chromosome differentiation in stickleback fishes. BMC Genomics 2011 12:474.

\section{Submit your next manuscript to BioMed Central} and take full advantage of:

- Convenient online submission

- Thorough peer review

- No space constraints or color figure charges

- Immediate publication on acceptance

- Inclusion in PubMed, CAS, Scopus and Google Scholar

- Research which is freely available for redistribution

Submit your manuscript at www.biomedcentral.com/submit 